E3S Web of Conferences 5, 04002 (2015)

DOI: $10.1051 /$ e3sconf/ 20150504002

(C) Owned by the authors, published by EDP Sciences, 2015

\title{
A study of wave forces on an offshore platform by direct CFD and Morison equation
}

\author{
D. Zhang ${ }^{1, a}$ and E. G. Paterson ${ }^{1, b}$
}

${ }^{1}$ Virginia Polytechnic Institute and State University, Blacksburg, Virginia, 24060, USA

\begin{abstract}
Engineering simulation tools for hydrodynamic loads on offshore platforms (e.g. NREL's HydroDyn and Orcina's OrcaFlex) use simplified theories and empirical models. The most common is the Morison's equation which accounts for unsteady drag and inertial forces created by incident waves. Coefficients are required for these models and are typically derived from wave-tank or pulsatile U-Shaped water tunnel experiments.

The first step of this study focuses on the application of Morison's equations for computing forces on a 3D platform using both computational fluid dynamics computed and empirical coefficients. Wave forces on a simple structure, such as a bottommounted monopile, are estimated by a strip-wise application of Morison's equation on each section. The fluid particles orbit and kinematics at strip-wise depth are readily derived from wave theory, and the total wave force on the supporting structure is computed by performing a simple integration in the vertical direction over each section.

The next step is the presentation of 3D multiphase RANS simulation of the wind-turbine platform in single-harmonic regular waves. Simulation results from full 3D simulation will be compared to the results from Morison's equation. We are motivated by the challenges of a floating platform which has complex underwater geometry (e.g. tethered semi-submersible). In cases like this, our hypothesis is that Morison's equation will result in inaccurate prediction of forces, due to the limitations of $2 \mathrm{D}$ coefficients of simple geometries, and that 3D multiphase RANS CFD will be required to generate reliable predictions of platform loads and motions.
\end{abstract}

\section{Introduction}

\subsection{Motivation}

Cyber Wind Facility (CWF) is a computational facility designed to generate data that cannot be collected from a field wind turbine facility, which has limited data collection capabilities (Brasseur et al., 2013). By using petascale computer systems, CWF can generate four-dimensional data over the entire domain in a controlled cyber environment, specifically including highly resolved 4-D cyber data;

\footnotetext{
a e-mail: liybzd@vt.edu

be-mail: egp@vt.edu
}

This is an Open Access article distributed under the terms of the Creative Commons Attribution License 4.0, which permits unrestricted use, distribution, and reproduction in any medium, provided the original work is properly cited. 
coupled wind-loading-torque data; coupled wave-motion-loadings data; test-bed, design and controls concepts and correlations for advanced design tools.

As a vital part of the whole project, the hydrodynamics module solves the 6-DOF equations of the tower-rotor-platform by applying hybrid URANS-LES around the platform coupled to a wave model with wide variety of wave characteristics. The resulting data can be used both in the design stage and performance calculation.

Current state-of-art engineering tools, for example NREL's Hydrodyn, OrcaFlex and ANSYS AQWA, are based upon semi-emperical time-domain methods, e.g., Cummins equation (Cummins, 1962). These models use various theories for radiation, diffraction, hydrostatics, and viscous effects. Morison's equation is commonly used for damping and inertia forces due to wave excitation.

For certain wave-structure configuration, for example the OC3 spar buoy platform (Jonkman, 2010), Morison's equation provides reasonable accuracy of wave forces on the floating structure. Large amount of historical data is available for simple geometries, e.g. circular cylinders. Some floating platforms have complex underwater geometry, e.g. OC4 Semi-submersible, which precludes the use of theoretical or historical data for drag, inertial, damping, added mass, etc.

\subsection{Objective}

There are two objectives of the current study:

\section{1) RANS CFD for fixed platform}

The objective of the current study includes performing multi-phase RANS simulations of a fixed platform in waves (i.e. the diffraction-wave problem), which is a precursor study prior to undertaking full $6 \mathrm{DOF} / \mathrm{RANS}$ simulations, including mooring-line models.

\section{2) Compare with results from Morison's equation}

The CFD result of wave-excitation forces for OC3 spar-buoy is then compared to Morison's equation model using both CFD-based or experimental coefficients.

\section{Cyber Wind Facility Hydrodynamic Module}

The hydrodynamic module in CWF is developed based on existing computational models including the wave-generating library. The computational models used in the hydrodynamic module includes:

1. Tightly coupled multiphase Navier-Stokes equations and Newton's 6DOF equation-of-motion. (Dunbar et al., 2014)

2. waves2Foam library for wave generation. (Jacobsen et al., 2012)

3. Catenary-line model for mooring-line forces. (Jonkman, 2007)

4. Selectable-fidelity model of the wind turbine, including actuator-disk and actuator-line models. ALM model developed by CWF team members. (Jha et al., 2013)

5. Dynamic meshing using Elliptic mesh-deformation model with variable stiffness, which maintains near-wall mesh quality. (Campbell et al., 2011) 


\section{1 waves2Foam library}

The waves2Foam library is a toolbox used to generate and absorb free surface waves via relaxation zones in arbitrary shape. Large range of wave theories are implemented together with corresponding boundary conditions for volume fraction, pressure and velocity field. Pre- an postprocessing utilities are provided for setting wave properties of a given wave field in the computational domain through dictionaries. (Jacobsen et al., 2012)

Common wave theories implemented in the waves2Foam library includes potential current; regular waves: Stokes 1st, 2nd and 5th order theory; solitary waves and irregular waves.

The boundary conditions in the library includes waveAlpha: FvPatchScalarField for the volume-of-fraction (VOF) ratio; wavePressure: FvPatchScalarField for the pressure gradient and waveVelocity: FvPatchScalarField for the velocity field.

\section{Relaxation techniques}

The relaxation techniques aim to eliminate the reflection and internally generated wave near boundaries. The relaxation zones provide numerical beaches for explicit and implicit damping of waves to control wave reflections. An explicit relaxation method is used in the study, which is performed after the momentum and volume-fraction equations are solved:

$$
\begin{aligned}
u & =(1-\omega) u_{\text {target }}+\omega u_{\text {computed }} \\
\alpha & =(1-\omega) \alpha_{\text {target }}+\omega \alpha_{\text {computed }}
\end{aligned}
$$

$\omega \in[0,1]$ is the weighting function. In the present library, the weighting function is given as (Jacobsen et al., 2012):

$$
\omega(\chi)=1-\frac{\exp \left(\chi^{3.5}\right)-1}{\exp (1)-1} \quad \text { for } \quad \chi \in[0,1]
$$

Variable $\chi$ is defined such that $\omega=1$ at the interface between relaxation zone and non-relaxed domain. As we approach the boundary, the velocity and VOF ratio reach target value according to the corresponding wave theory (Figure 1).
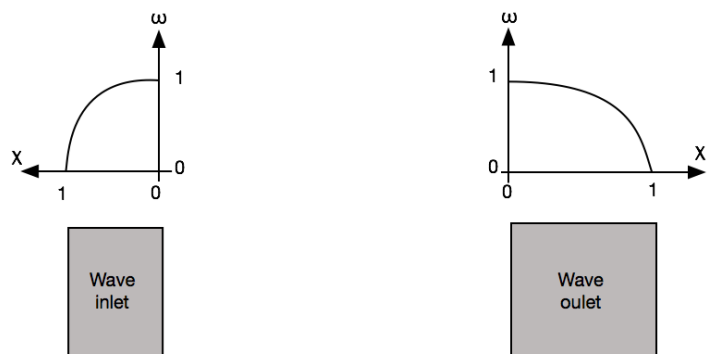

Figure 1: Variation of the weighting function in wave inlet and outlet relaxation zone

\section{Utilities}

Both pre- and postprocessing utilities are provided, some of them are very useful and can reduce computing time in some cases. For example, setWaveField, which is similar to setFileds, it sets the initial condition in the computational domain including velocity and alpha (VOF ratio) fields. 
E3S Web of Conferences

\begin{tabular}{|c|c|c|}
\hline & Wave Height(H) & Wave Length(L) \\
\hline Base Case & $3 m$ & $60 m$ \\
\hline Case 2 & $3 m$ & $90 m$ \\
\hline Case 3 & $7 m$ & $90 m$ \\
\hline Case 4 & $0.65 m$ & $90 m$ \\
\hline
\end{tabular}

Table 1: Four-case setup

\section{Flow Conditions and Floating Platform Geometry}

\subsection{Flow Conditions}

The flow conditions used in this study are based on the statistical data from National Data Buoy Center (NDBC). Station 44014 near Virginia Beach is chosen since it's close to the research area of Virginia Offshore Wind Technology Advancement Project (VOWTAP), which is sponsored by Department of Energy (DOE) for initial engineering design of offshore wind facility.

From the NDBC, the real time wave data is updated every 1 hour and historical data in the last two decades are also provided. This data includes standard meteorological data, continuous wind data, spectral wave data and ocean current data. Figure 2 and 3 shows an example of the spectral density and significant wave height from NDBC's database.

Both the flow conditions and geometry of the structure determines the dominant physics, hence we need different models to approximate the hydrodynamic force. In Figure $4, H$ is the wave height, $D$ is the circular cylinder diameter and $L$ is the wave length. The vertical axis $H / D$ gives relative wave height, which determines the magnitude of drag compared to inertial force; the horizontal axis $\pi D / L$ is defined as the diffraction coefficient, which gives the importance of diffraction effects.

Morison's equation excludes the diffraction effects and divides total force into inertial and drag force. $C_{m}$ and $C_{d}$ are the inertial and drag coefficient respectively in Figure 4, and Morison's equation is applicable in region I, III, V and VI. Four cases indicated by red dots are computed in this study, they fall in region I and III. The respective wave height and wave length are listed in table 1, for simplicity, all waves are calculated by linear theory.

\subsection{Floating Platform Geometry}

\section{OC3 spar buoy}

OC3-Hywind spar buoy as shown in Figure 5 is studied here. The whole platform is $130 \mathrm{~m}$ in length, $6.5 m$ in diameter before and $9.4 m$ after taper (Jonkman, 2010). As a preliminary study, no mooring line is attached to the buoy and the whole structure is fixed in water. Furthermore, only a single harmonic of the ocean wave is simulated. A full-scale mesh is built around the buoy for CFD simulation and Morison's equation is applied to each section of the buoy according the the theoretical particle velocity and structure diameter.

\section{Semi-submersible}

Eventually we want to extend our calculation to more complex structure, for example, the semisubmersible platform with mooring lines(Robertson, et al., 2013) shown in Figure 6. Full wave spectrum will be added into the simulation together with the aerodynamic force on the turbine. Dunbar, Craven and Paterson (2014) have been using the semi-submersible for validation of the tightly-coupled RANS/6DOF algorithm and for testing of dynamic meshing strategies of complex geometries. 


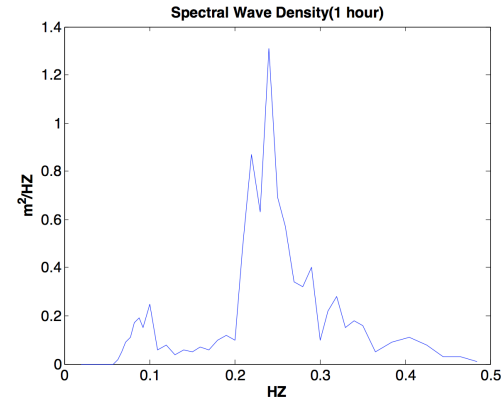

Figure 2: Spectral wave density in 1 hour

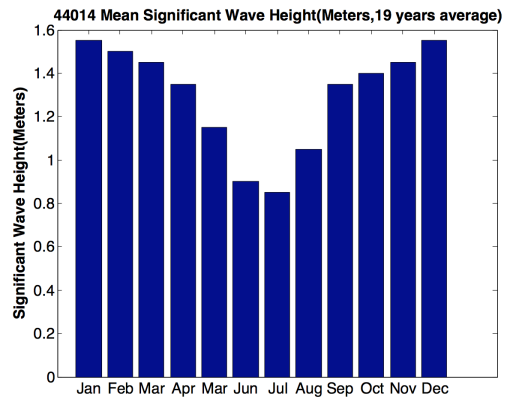

Figure 3: Mean significant wave height for each month in 19-year

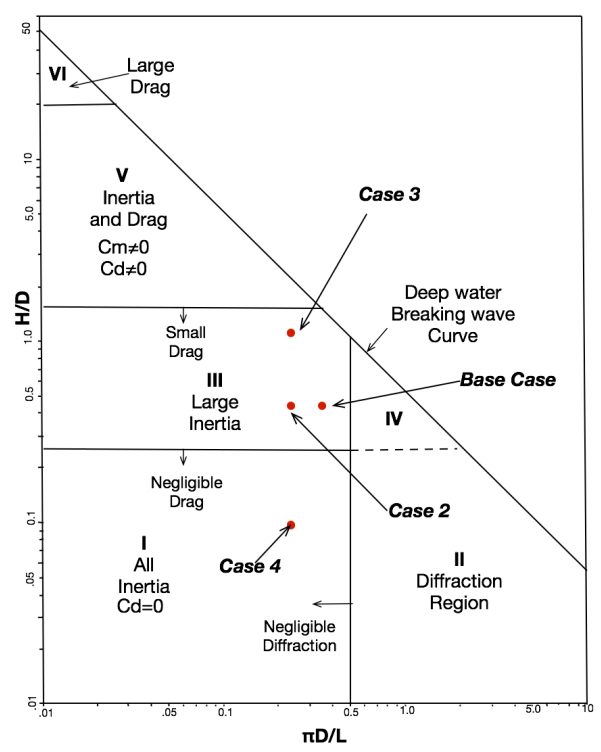

Figure 4: Summary of 4 cases and corresponding dominant physic

\section{CFD Simulations}

\subsection{Case setup}

A full-scale mesh is generated using Pointwise. The domain spans from $-120 \mathrm{~m}$ to $120 \mathrm{~m}$ in $\mathrm{x}-$ direction, i.e. the wave propagation direction; $-60 m$ to $60 m$ in y-direction or the spanwise direction; in $\mathrm{z}$-direction, the domain reaches $-180 \mathrm{~m}$ under and $120 \mathrm{~m}$ above water line to accommodate both the tower and the platform. Future simulations will need to extend the domain further into the atmosphere to adequately resolve the turbine rotor disc. The schematics of the domain is shown in Figure 7. Furthermore, the mesh is clustered near cylinder wall and design waterline (DWL). Uniform axial 


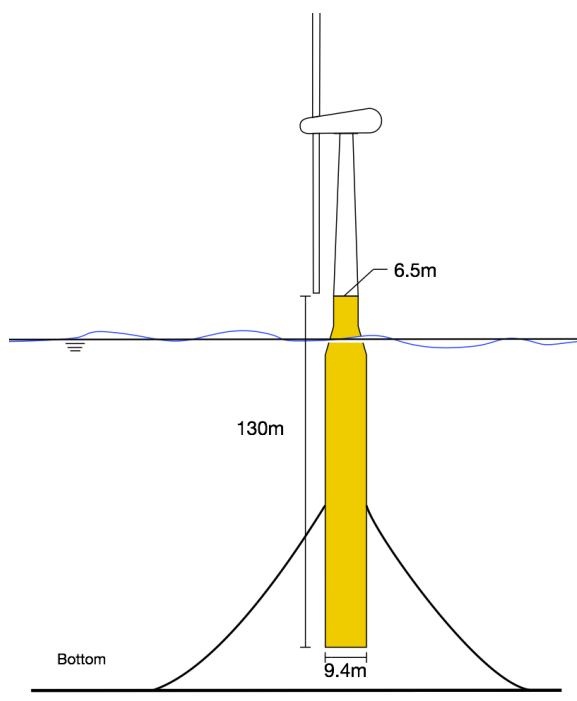

Figure 5: Schematics of OC3-Hywind spar buoy with wind turbine in ocean waves and currents

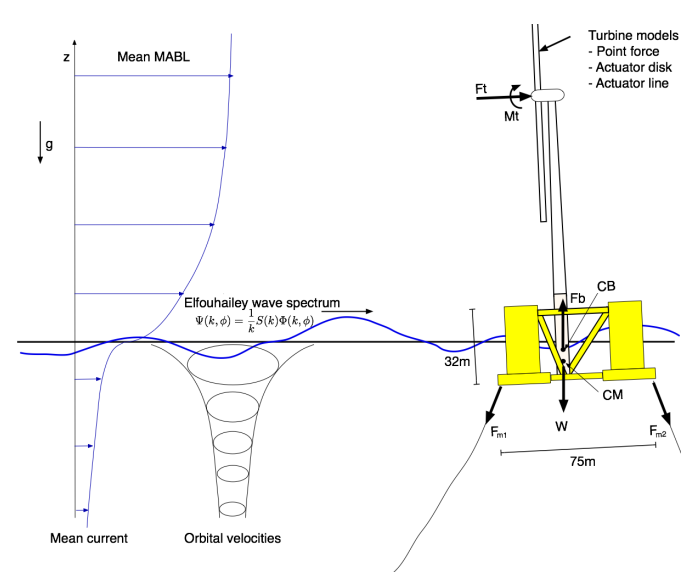

Figure 6: Schematics of Semi-Submersible platform with wind turbine in ocean waves and currents

spacing mesh upstream of buoy is generated for resolving waves and the boundary conditions are shown in Figure 8.

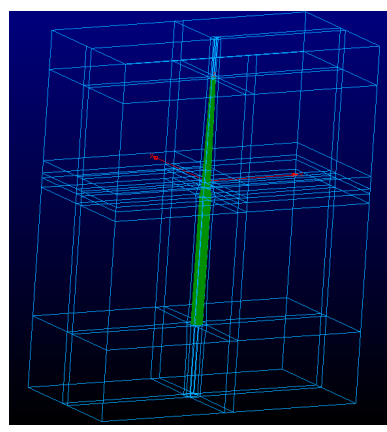

Figure 7: Schematics of the computation domain

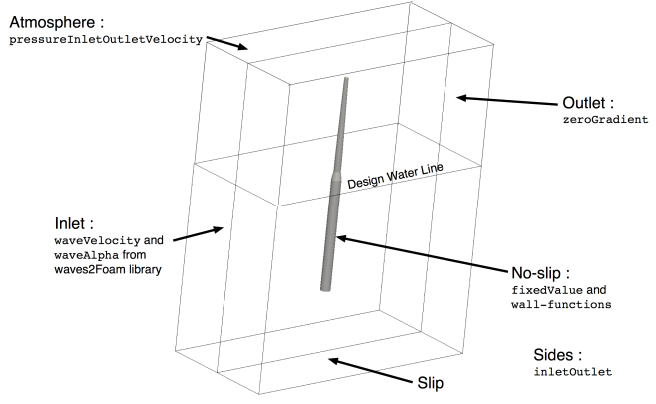

Figure 8: Boundary conditions of the computation filed

The computational parameters are set to achieve both high stability and fast convergence. For FV schemes, celllimited Gauss linear is used for gradSchemes of $U$ and alpha1 and in divSchemes subdictionary the convection schemes used includes: 1) Gauss linearUpwindV for momentum equation; 2) Gauss vanLeer for VOF equation; 3) Gauss interfaceCompression for interface sharpening; 4) Gauss upwind for turbulence models. In FV solvers, we have GAMG with the DIC smoother for pressure-Poisson equation and PBiCG for all other equations. Finally, set- 
tings in PISO algorithm control are: 1) nCorrectors 3; nNonOrthogonalCorrectors 1; nAlphaCorr 1; nAlphaSubCycles 1; cAlpha 1.

\subsection{HPC resources}

The Advanced Research Computing (ARC) center at Virginia Tech provides High-Performance Computing (HPC) resource for all CFD simulations. Two of the Top-500 clusters are used in this study: BlueRidge, 408-node Cray CS-300 cluster, each node is outfitted with two octa-core Intel Sandy Bridge CPUs and 64GB memory; Hokiespeed, GPU-accelerated cluster with 204 nodes. Each nodes has 24GB memory, two six-core Xeon E5645 CPUs with two NVIDIA M2025/C2050. Each user can request up to 1024 cores on Blueridge and 384 cores on Hokiespeed and the maximum run time in normal queue is 144 hours and 72 hours respectively.

While we have OpenFOAM 2.2 and 2.3 installed on our cluster, foam-extend-3.0 is our preferred version due to the community-focused development model. For a mesh of $5 \mathrm{M}$ cells, execution time is less than 3 hours on 96 processors for 120s of physical time (approx. 10-15 wave encounters).

\subsection{CFD results}

The free-surface wave elevation contours are shown in Figure 9 and 10. In Figure 9, we can observe diffraction waves around the buoy and Figure 10 shows the wave-air interaction, which is also an interesting topic. The exponential decrease in velocity magnitude with depth is shown in Figure 11. For reference, velocity in $\mathrm{x}$ - and $\mathrm{z}$-direction of 1 st-order Stokes wave is expressed as

$$
\begin{aligned}
& \left.u(x, t)=\omega A e^{k z} \cos (k x-\omega t)\right) \\
& \left.w(x, t)=\omega A e^{k z} \sin (k x-\omega t)\right)
\end{aligned}
$$

$z$ is the water depth (negative) and $\omega, k$ and $A$ are the wave angular speed, wavenumber and amplitude.

The unsteady inline force (x-direction) history of four cases are shown in Figure 12, four cycles of wave force history are plotted after the initial transient. From the plot we observe that time history does not display perfect limit-cycle behavior after 9 periods. Also it's not perfect sinusoid which is expected for both inertia and drag.

\section{Morison's Equation and Strip Theory}

\subsection{Morison's equation}

Morison's equation for circular cylinder is expressed as:

$$
\begin{aligned}
F & =\frac{\pi}{4} C_{m} \rho D^{2} \frac{\partial U}{\partial t}+\frac{1}{2} C_{d} \rho D U|U| \\
U & =U_{m} \sin (\omega t)
\end{aligned}
$$

$F$ is the inline force; $C_{m}$ and $C_{d}$ are the inertial and drag coefficients. Both $C_{m}$ and $C_{d}$ are functions of Keulegan-Carpenter number $K=U_{m} T / D$, where $T$ is the period of the oscillatory flow, and Reynolds number $R e=U_{m} D / v ; U_{m}$ is the maximum velocity in one cycle. As an empirical approach, a vast 


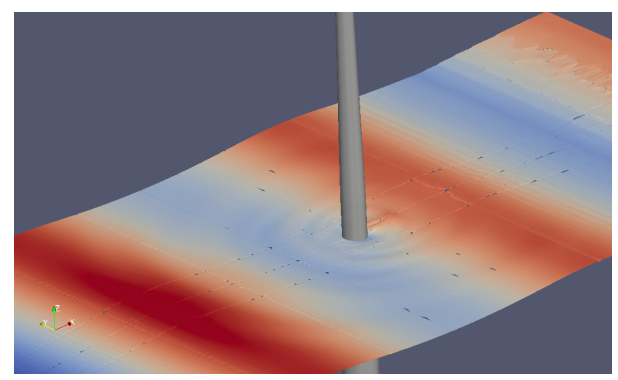

Figure 9: Wave elevation on free surface $(H=3.5 m, L=90 m)$

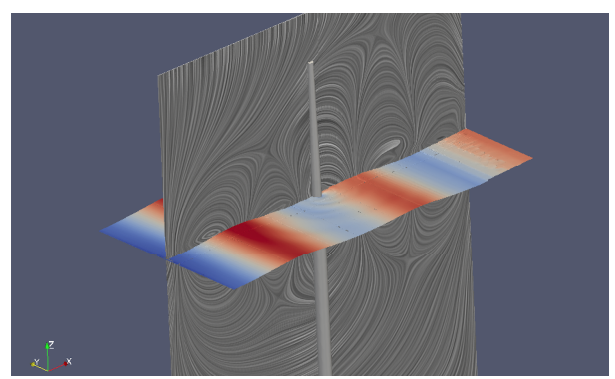

Figure 10: Wave elevation on free surface with wave-induced circulations in $\mathrm{y}$ plane $(H=3.5 m, L=90 m)$

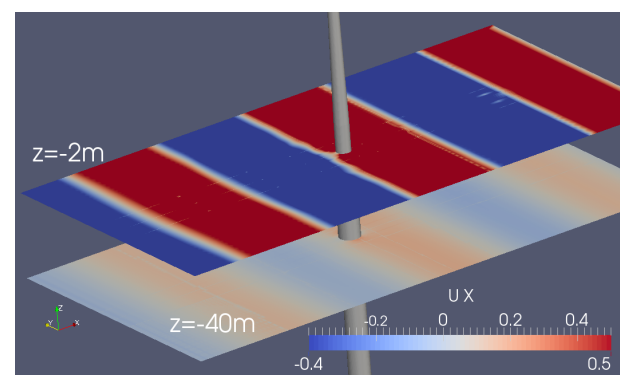

Figure 11: Variation of flow velocity in $\mathrm{x}$ direction with depth $(H=3.5 m, L=90 m)$

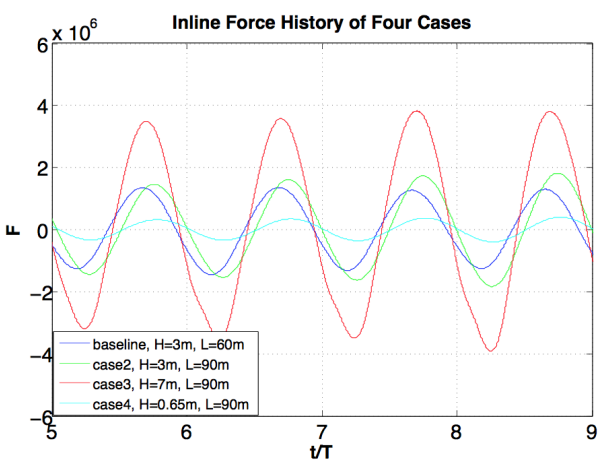

U-Shape water tunnel (Sarpkaya 1976)

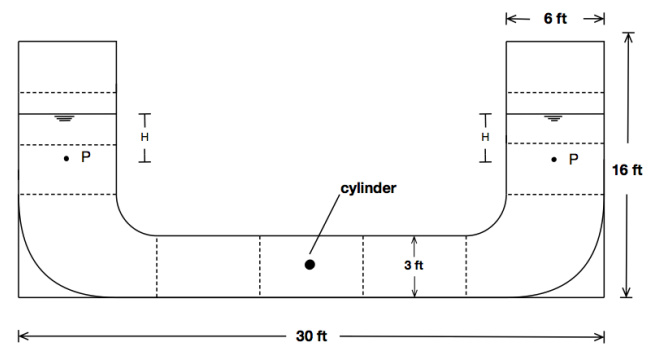

Figure 13: Schematics of U-Shape tunnel (Sarp-

Figure 12: Inline force history of four cases kaya [1976])

range of experimental data on $C_{m}$ and $C_{d}$ is available for numerous lab and field tests, it provides acceptable and reliable prediction of wave force on many offshore structures.

Sarpkaya (1976a) conducted a series of experiments in a U-shape water tunnel to study the hydrodynamic force on different size cylinders in various flow conditions. The apparatus of the experiments are shown in Figure 13, the period of the sinusoidal motion is $5.5 \mathrm{~s}$, seven cylinders with 
diameter ranging from 2 to 6.5 inches were used.

Figure 14 and 15 show part of the result from Sarpkaya's experiments, the frequency parameter $\beta$ is defined as

$$
\beta=\operatorname{Re} / K=\frac{D^{2}}{v T}
$$

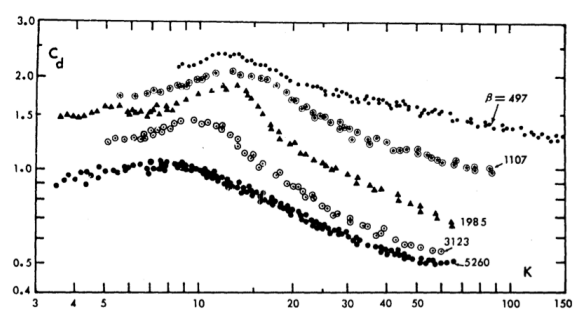

Figure 14: $C_{d}$ versus $\mathrm{K}$ for various values of $\beta$ (Sarpkaya [1976])

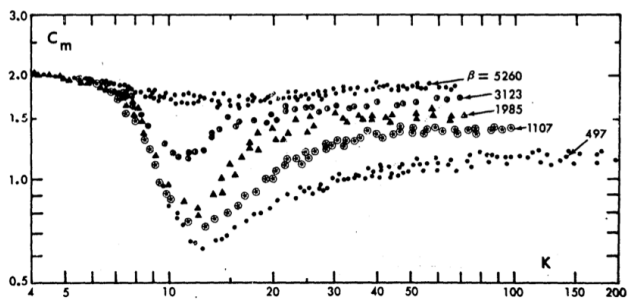

Figure 15: $C_{m}$ versus $\mathrm{K}$ for various values of $\beta$ (Sarpkaya [1976])

In full-scale cases, the flow around floating platform has very high Reynolds number, several magnitudes higher than the experiments, Sarpkaya (1976b) also studied the force on cylinder at high Reynolds number.

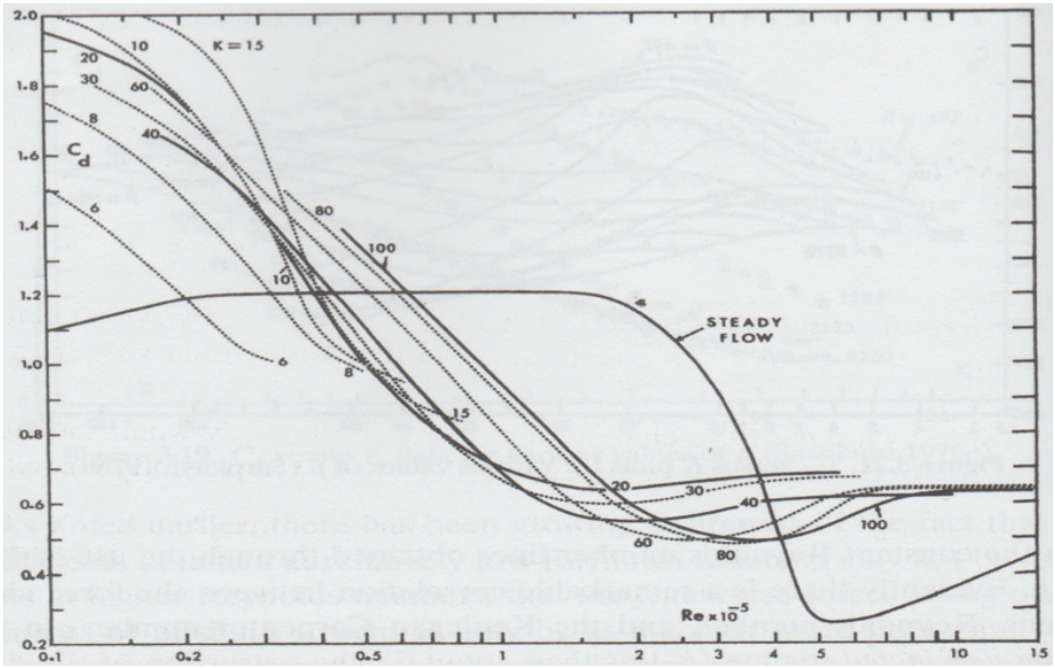

Figure 16: $C_{d}$ versus $\operatorname{Re}$ for various values of $\beta$ (Sarpkaya[1976])

Figure 16 and Figure 17 shows that at high $\mathrm{Re}, C_{m}$ approaches 1.8 and $C_{d}$ approaches 0.65 at various values of $\mathrm{K}$. In reality, Reynolds number along the vertical length of the pile ranges from $10^{7}$ near free surface to $10^{5}$ at wave base, we can safely assume that

$$
C_{m}=1.8 \quad \text { and } C_{d}=0.65
$$




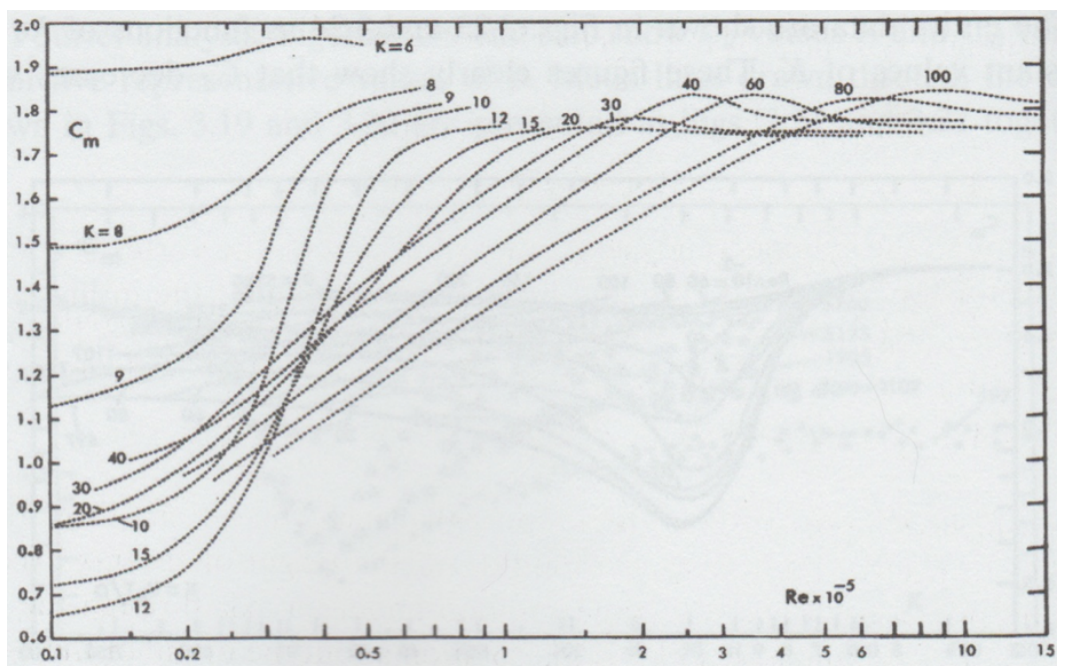

Figure 17: $C_{m}$ versus Re for various values of $\beta$ (Sarpkaya[1976])

\subsection{Strip theory}

Application of Morison's equation by strip theory is based on the fact that water particle velocity at various depth is readily derived from linear wave theory. As mentioned above, we assume $C_{m}=1.8$ and $C_{d}=0.65$ in Morison's equation, according to the geometry of the spar buoy, we apply Morison's equation to each strip as shown in Figure 18 and then integrate along the depth to derive the total wave force.

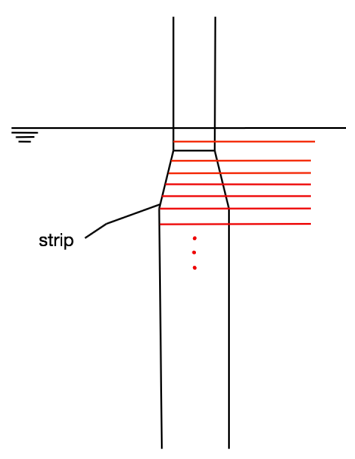

Figure 18: Strips along the depth

By assuming linear theory, we have the ratio between inertial and drag force:

$$
\frac{f_{D_{\max }}}{f_{I_{\max }}}=\frac{C_{d}}{\pi^{2} C_{m}} K \approx 0.036 K
$$




\begin{tabular}{|c|c|c|c|c|}
\hline & Base & Case 2 & Case 3 & Case 4 \\
\hline CFD $(N)$ & $1.3 e 6$ & $1.6 e 6$ & $3.5 e 6$ & $3.5 e 5$ \\
\hline Morison's equation $(N)$ & $1.25 e 6$ & $1.4 e 6$ & $3.3 e 6$ & $3.1 e 5$ \\
\hline Comparison Error \% & 3.8 & 12.5 & 5.7 & 11.4 \\
\hline
\end{tabular}

Table 2: Peak force comparison

By calculating the variation of the $\mathrm{K}$ in different depth and assuming, for example, $H=1.5 \mathrm{~m}$, even for the highest K in Figure 19, we have $\frac{f_{D_{\max }}}{f_{\max }}=5.3 \%$. As shown in Figure 20, it's clear that drag force is negligible compared to inertial force.

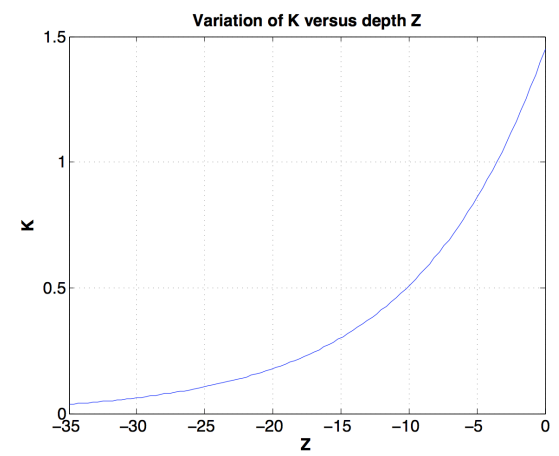

Figure 19: Exponential variation of $\mathrm{K}$ with depth Z

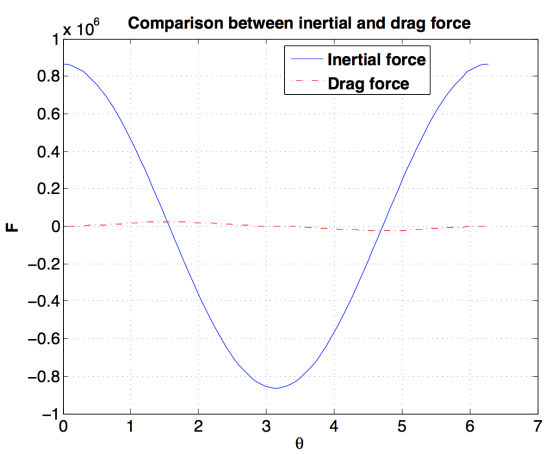

Figure 20: Inline force history for $H=1.5 m$ in one cycle

\section{Comparison}

The peak force from strip theory is readily derived from the above equation with assumption on force coefficients. The comparison between strip theory and CFD is given in table 2 .

In every case, the CFD peak force is larger than Morison's equation by $3 \%$ to $13 \%$. Physically, Morison's equation excludes the effect of diffraction, while from Figure 9 we can observe the diffraction wave. Numerically, there are two additional possible reasons: 1) Lack of full-scale coefficients and the uncertainty of extrapolation from model scale. Since inertial force is dominant as shown in Figure 20, it is very important to investigate the inertial coefficient $C_{m}$ for full-scale case by CFD simulations. 2) Numerical uncertainties in CFD and lack of grid-independence study. The refinement of the mesh, especially near-wall resolution, is relatively low. We need to address the above issues before repeating the simulation for complex geometries.

\section{Conclusion}

The "Cyber-Wind Facility" Hydrodynamic Module has been used for several different applications:

- 2D simulation of KC problem for Morison's equation coefficients at model scale for comparison to Sarpkaya (1976a)[not discussed] 
- Simulation of OC3 spar-buoy in waves using interFoam and waves2Foam. Conditions set to $H_{S}$ typical for future offshore-wind-plant near the coast of Virginia.

CFD simulations predict higher average peak force than Morison's equation. The hypothesis for the discrepancy is that diffraction effects is neglected in Morison's equation, full-scale coefficients are unknown, CFD accuracy not yet assessed using domain-size, time-step and grid refinement studies. However, the use of robust HPC resources at VT-ARC give good turn-around, many cases can be simulated in 24-hour period.

\section{Future work}

As a preliminary study of the module, there are many aspects need to be explored in the future, including re-mesh for large domain which includes rotor disc; compute Morsion's equation coefficients at full-scale conditions, and quantify 3D and scale effects; test and debug mooring-line model; collaborate with CWF team members: 1) Use tightly coupled 6DOF/RANS solver (Dunbar et al., 2014) and 2) Incorporate turbine forces (Jha et al., 2013).

\section{References}

[1] Brasseur, J., Paterson, E., Schmitz, S., Campbell, R., Vijayakumar, G., Lavely, A., ... \& Haupt, S. 2013 A “Cyber Wind Facility” for HPC Wind Turbine Field Experiments, In APS March Meeting Abstracts (Vol. 1, p. 1189).

[2] Cummins, W. 1962. The impulse response function and ship motion, David Taylor Model BasinDTNSRDC

[3] Campbell, R. \& Paterson, E. 2011. Fluid-structure interaction analysis of flexible turbomachinery, Journal of Fluids and Structures 27(8)

[4] Dunbar, A., Craven, B. \& Paterson, E. 2014. Application of a Tightly-Coupled CFD/6-DOF Solver for Simulating Offshore Wind Turbine Platforms, 2nd SOWE Presentation

[5] Jacobsen, N., Fuhrman D \& Fredsøe, J. 2012. A wave generation toolbox for the open-source CFD library: OpenFoam $\AA$

[6] Jha, P., Churchfield, M., Moriarty P., \& Schmitz, S. 2013. Accuracy of State-of-the-Art ActuatorLine Modeling for Wind Turbine Wakes, 51st AIAA Aerospace Sciences Meeting

[7] Jonkman, J. 2010. Definition of the Floating System for Phase IV of OC3, Technical Report: NREL/TP-500-47535, National Renewable Energy Laboratory

[8] Jonkman, J. 2007. Dynamics Modeling and Loads Analysis of an Offshore Floating Wind Turbine, Technical Report: NREL/TP-500-41958, National Renewable Energy Laboratory

[9] Robertson, A., Jonkman, J., Musial, W., Vorpahl, F. \& Popko, W. 2013. Offshore Code Comparison Collaboration, Continuation: Phase II Results of a Floating Semisubmersible Wind System, Technical Report: NREL/CP-5000-60600, National Renewable Energy Laboratory

[10] Sarpkaya, T. 1976a. In-line and Transverse Forces on Smooth and Sand-roughened Cylinders in Oscillatory Flow at High Reynolds Numbers, National Science Foundation

[11] Sarpkaya, T. 1976b. Vortex Shedding and Resistance in Harmonic Flow about Smooth and Rough Circular Cylinders at High Reynolds Numbers 